\title{
Dynamic STRess InTensity FaCtor for Subsurface Inclined Cracks
}

\author{
By Chien-Ching $\mathrm{Ma}^{1}$ and Szu-Kuzi Chen ${ }^{2}$
}

\begin{abstract}
To gain insight into the phenomenon of the interaction of stress waves with material defect, the transient problem of a half-space containing a subsurface inclined semi-infinite crack subjected to dynamic antiplane loading on the boundary of the half-space is investigated in this study. The solutions are determined by superposition of the fundamental solution in the Laplace transform domain. The fundamental solution is the exponentially distributed traction on crack faces. The exact close form transient solutions of dynamic stress intensity factor are expressed explicitly in time domain. These solutions are valid for an infinite length of time and have accounted for the contributions coming from incident, reflected and diffracted waves. Numerical results of dynamic stress intensity factors are obtained and compared with the corresponding static values. The transient solution is shown to approach the static value after the first few waves interactive with the crack tip have passed.
\end{abstract}

\section{INTRODUCTION}

The difficulty in determining the transient stress field in a crack-elastic body subjected to dynamic loading is well known. A considerable amount of research has been directed towards the solution of problems involving interaction of stress waves with crack and boundary to improve an understanding of the behavior of material failure under dynamic loading. In conventional studies of a semi-infinite crack in an unbounded medium subjected to a uniformly distributed dynamic loading on crack faces, the complete solution is obtained by integral transform methods together with direct application of the Wiener-Hopf technique (Noble 1958) and the Cagniardde Hoop method (de Hoop 1958) of Laplace inversion. If the loading is replaced by a nonuniform distribution having a characteristic length, then the same procedure using integral transformation methods does not apply. The problem of an elastic solid containing a semi-infinite crack subjected to concentrated impact loading on the faces of the crack has been previously studied by Freund (1974). He proposed a fundamental solution arising from an edge dislocation climbing along the line ahead of the crack tip with a constant speed to overcome these difficulties of the case with a characteristic length. The solution can be constructed by taking an integration over a climbing dislocation of different moving velocity. A number of problems for a semi-infinite crack subjected to impact loading have been analyzed by Brock $(1982,1984,1985)$ and Ma and Hou $(1990,1991)$ by using the method provided by Freund (1974). Recently, Lee and Freund (1990) analyzed fracture initiation of an edge cracked plate subjected to an asymmetric impact.

Whenever dynamic loading is applied to a body with an internal crack,

${ }^{1}$ Prof., Dept. of Mech. Engrg., Nat. Taiwan Univ., Taipei, Taiwan, 10764, Republic of China.

${ }^{2}$ Grad. Student, Dept. of Mech. Engrg., Nat. Taiwan Univ., Taipei, Taiwan 10764, Republic of China.

Note. Discussion open until August 1, 1994. To extend the closing date one month, a written request must be filed with the ASCE Manager of Journals. The manuscript for this paper was submitted for review and possible publication on January 25, 1993 . This paper is part of the Journal of Engineering Mechanics, Vol. 120, No. 3, March, 1994. CASCE, ISSN 0733-9399/94/0003-0483/\$2.00 + \$.25 per page. Paper No. 5489 . 
the resulting stress waves may initiate crack growth. Few solutions for a cracked elastic solid subjected to dynamic loading are available. Exact transient close form solutions for a stationary semi-infinite crack subjected to a suddenly applied dynamic body force in an unbounded medium have been obtained by Tsai and Ma (1992) for inplane case and by Ma and Chen (1993) and Brock (1986) for antiplane case. Brock (1983) investigated the dynamic stress intensity factor for a stationary semi-infinite crack due to the arbitrary motion of a screw dislocation in an unbounded medium.

Tsai and Ma (1993) studied the dynamic response of the stress intensity factor of an inclined semi-infinite crack inside an elastic half-plane and subjected to dynamic impact inplane loading on the half-plane. Brock et al. (1985) investigated the case in which the crack is normal to the halfplane surface and the point loading is applied to the surface directly above the crack tip, which is a special case of the general formulation by Tsai and Ma (1993). All the results mentioned above are valid only before the first wave scattered from the crack tip returns to the crack tip after being reflected by the boundary. The problem to be considered in this study is the antiplane response of an elastic half-plane, with an inclined crack extending from infinity to the location near the half-plane surface, which is subjected to dynamic antiplane loading on the half-plane surface as shown in Fig. 1. Particular attention is given to the elastic field near the crack tip, which is completely characterized by the stress intensity factor.

The investigation of an idealized semi-infinite crack can provide some information for a realistic elastodynamic fracture problem. It is noted that while the analysis has been carried out assuming a semi-infinite crack, the results remain valid for a finite crack up until the time at which waves diffracted from the far tip reach the tip near the boundary. A pre-existing fault inside the medium would disturb the propagation waves and make the theoretical analysis much more difficult than in an homogeneous medium. In this study, half-plane boundary and crack are involved in the analysis which will make the analysis extremely difficult. In analyzing this problem, the reflections and diffractions of stress waves by the boundary and by the crack must be taken into account, which will generate infinite waves. This problem involves a characteristic length, which is the distance from the

\section{$(l, h)$}

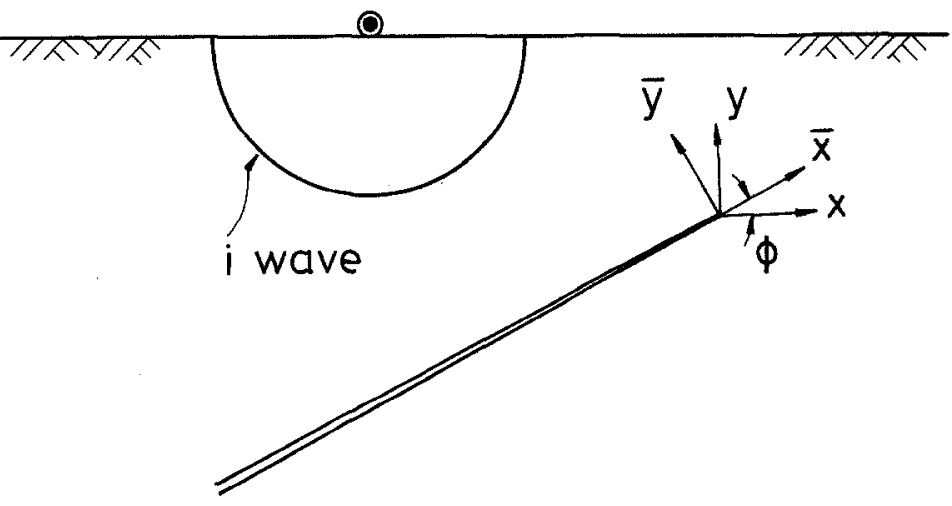

FIG. 1. Configuration and Coordinate Systems of Subsurface Inclined Crack Subjected to Impact Loading on Half-Space 
applied loading to the crack tip and makes a direct solution by standard techniques difficult. Furthermore, none of the methods proposed by Freund (1974) and Brock et al. (1985) have worked for this problem. Therefore, some other approach must be followed. A new fundamental solution is used for overcoming these difficulties. This alternative fundamental solution is successfully applied towards solving the problem and is to be demonstrated to become an efficient methodology. The stress intensity factor is the key parameter in characterizing fracture initiation and we will focus our attention on the evaluation of the dynamic stress intensity factor. The explicit closedform formulation of the dynamic stress intensity factor is obtained and the dynamic effect of each wave is presented in this study. The results are valid for an infinite number of waves that are scattered from crack tip and reflected by the half-plane boundary. In some classes of dynamic problems, the ability to find a static field may hinge on waiting for the wave fronts to pass and the transient effect to die away. The characteristic time after which the transient effect can be neglected is also investigated in this study.

\section{BASIC FUNDAMENTAL SOLUTIONS}

As usual in problems of the type considered here, superposition of solutions plays a significant role. Most of the solutions on scattering of elastic waves by cracks in solids are based on the theory of superposition and carried out in Fourier or Laplace transformed domain. There are some papers that studied the problem of scattering of elastic waves from an interface crack in a layered medium (Yang and Bogy 1985; Boström 1987) or two halfspaces (Srivastara et al. 1978; Kundu 1988). The solutions to the problem considered in this study can be determined by superposition of the following problems. Problem A treats the dynamic concentrated force acting on the half plane medium without a crack, inducing a traction on the planes that is to eventually define the crack faces. In problem B, an infinite body containing a semi-infinite crack is considered in which the faces are subjected to tractions that are equal and opposite to those on the corresponding planes in problem $\mathrm{A}$. Problem $\mathrm{C}$ considers a half-plane free surface subjected to incident waves, which is the disturbance generated by the crack in problem B. The reflected waves coming from the free boundary can be constructed by employing the method of images. The sum of the solutions to problems $\mathrm{A}, \mathrm{B}$ and $\mathrm{C}$ is the solution of the problem considered in this study. Problem $B$ in the aforementioned three fundamental problems is the only one that needs careful analysis.

From physical considerations, reflected and diffracted waves are generated in order to eliminate the stress induced by incident waves on the traction-free boundaries. For most of the dynamic problems, the incident waves can be represented in an exponential functional form in the Laplace transform domain of time. The reflected and diffracted waves generated by the crack can thus be constructed by the superposition method, that is, if the responses toward an applied exponentially distributed traction on the crack face in the Laplace transform domain can be obtained preliminarily.

Consider the fundamental problem of antiplane deformation for a semiinfinite crack contained in an unbounded medium. The solution for an exponentially distributed loading applied at the crack faces in the Laplace transform domain is denoted as the fundamental solution. The problem can be viewed as a half-plane problem 'with the material occupying the region $y \geq 0$, subjected to the following mixed boundary conditions in the Laplace transform domain 
$\bar{\tau}_{y z}(x, 0, p)=e^{p \eta x} \quad(-\infty<x<0)$

$\overline{\mathrm{w}}(x, 0, p)=0 \quad(0<x<\infty)$

where $p=$ the Laplace transform parameter and $\eta=$ a constant. The overbar symbol is used for denoting the transform on time $t$. This fundamental problem has no characteristic length and can be easily solved by standard transform and Wiener-Hopf techniques. The governing equation can be represented by the two-dimensional wave equation

$\frac{\partial^{2} w}{\partial x^{2}}+\frac{\partial^{2} w}{\partial y^{2}}=b^{2} \frac{\partial^{2} w}{\partial t^{2}}$

where $b=$ the slowness of the transverse wave given by

$b=\frac{1}{v}=\sqrt{\frac{\rho}{\mu}}$

in which $w(x, y, t)=$ the displacement normal to the $x y$-plane; $v=$ the shear wave speed, and $\mu$ and $\rho=$ the respective shear modulus and the mass density of the material. The nonvanishing shear stresses are

$$
\begin{aligned}
& \tau_{y z}=\mu \frac{\partial w}{\partial y} \\
& \tau_{x z}=\mu \frac{\partial w}{\partial x}
\end{aligned}
$$

This problem can be solved by the application of integral transforms. Applying the one-sided Laplace transform over time, the two-sided Laplace transform over $x$ under the restriction of $\operatorname{Re}(\eta)>\operatorname{Re}(\lambda)$ and the WienerHopf technique is implemented. The solutions of stresses and displacement in the transform domain for the boundary conditions 1 and 2 are

$$
\begin{aligned}
& \bar{\tau}_{y z}(x, y, p)=\frac{1}{2 \pi i} \int_{\Gamma_{\lambda}} \frac{(b+\lambda)^{1 / 2} e^{-p(\alpha y-\lambda x)}}{(b+\eta)^{1 / 2}(\eta-\lambda)} d \lambda \ldots \ldots \ldots \ldots \\
& \bar{\tau}_{x z}(x, y, p)=-\frac{1}{2 \pi i} \int_{\Gamma_{\lambda}} \frac{\lambda e^{-p(\alpha y-\lambda x)}}{(b+\eta)^{1 / 2}(\eta-\lambda)(b-\lambda)^{1 / 2}} d \lambda \ldots \\
& \bar{w}(x, y, p)=-\frac{1}{2 \pi i} \int_{\Gamma_{\lambda}} \frac{e^{-p(\alpha y-\lambda x)}}{\mu p(b+\eta)^{1 / 2}(\eta-\lambda)(b-\lambda)^{1 / 2}} d \lambda
\end{aligned}
$$

where

$\alpha=\left(b^{2}-\lambda^{2}\right)^{1 / 2}$

The corresponding result of the dynamic stress intensity factor in the Laplace transform domain is

$$
\begin{aligned}
& \bar{K}(p)=\lim _{x \rightarrow 0} \sqrt{2 \pi x} \bar{\tau}_{y z}(x, 0, p) \\
& \bar{K}(p)=-\frac{\left(\frac{2}{\pi}\right)^{1 / 2} \Gamma\left(\frac{1}{2}\right)}{(b+\eta)^{1 / 2} p^{1 / 2}} \ldots .
\end{aligned}
$$




\section{EXPLICIT TRANSIENT SOLUTIONS OF DYNAMIC STRESS INTENSITY FACTORS}

The investigation of a subsurface crack subjected to dynamic loading is an important topic in material failure analysis. The problem considered here is an inclined semi-infinite crack located under the surface of a half-plane. We focus our attention mainly on the dynamic stress intensity factor in this study. Having two coordinate systems in the following analysis is convenient since the surface of the half plane is not parallel to the crack faces. The origins of the two coordinate systems $(\bar{x}, \bar{y})$ and $(x, y)$ are both located at the crack tip, as shown in Fig. 1. The planar crack lies in the plane $\bar{y}=0$, $\bar{x}<0$ and the inclined angle of the crack is $\phi$. The valid range of $\phi$ for this analysis is $0 \leq \phi \leq \pi / 2$. The coordinate transforms and stress relations between these two systems are

$\bar{x}=x \cos \phi+y \sin \phi$

$\bar{y}=-x \sin \phi+y \cos \phi$

$\tau_{x z}=\tau_{\overline{x z}} \cos \phi-\tau_{\overline{y z}} \sin \phi$

$\tau_{y z}=\tau_{\overline{x z}} \sin \phi+\tau_{\overline{y z}} \cos \phi$

This problem has a characteristic length. A direct attempt towards solving this problem by Wiener-Hopf techniques is not applicable. The transient elastodynamic problem is solved by superposition of the fundamental solutions obtained in the previous section in the Laplace transform domain. The transient solutions are composed of an incident field, reflected field, and diffracted field, which are denoted by superscripts of $i, r$, and $d$, respectively. The incident wave is the response to loading applied to a semiinfinite unbounded medium. The reflected and diffracted waves are generated through application of an opposite traction at the crack surface to eliminate the stress induced by incident wave.

Consider a half-plane body that is stress-free and at rest. At time $t=0$, an antiplane concentrated dynamic force is applied at the surface of a half plane at the position $x=l, y=h$, as shown in Fig. 1 . The time dependence of the loading is represented by the Heaviside step function $H(t)$. The incident fields of shear stresses in the Laplace transform domain can be expressed as follows

$$
\begin{aligned}
& \tau_{y z}^{i}(x, y, p)=\frac{1}{2 \pi i} \int_{\Gamma_{\lambda}} e^{p[\alpha(y-h)+\lambda(x-l)]} d \lambda \\
& \tau_{x z}^{i}(x, y, p)=\frac{1}{2 \pi i} \int_{\Gamma_{\lambda}} \frac{\lambda}{\alpha} e^{p[\alpha(y-h)+\lambda(x-l)]} d \lambda
\end{aligned}
$$

The incident stress field $\bar{\tau}_{\bar{y} \bar{z}}^{i}(\bar{x}, 0, p)$ induced in the crack face at $\bar{y}=0$ expressed in the $\bar{x}-\bar{y}$ coordinate system is

$\bar{\tau} \overline{y z}(\bar{x}, 0, p)=\frac{1}{2 \pi i} \int_{\Gamma_{\lambda}} e^{-p \alpha h_{0}+p \lambda\left(\bar{x}-l_{0}\right)} d \lambda$

where

$$
\begin{aligned}
& l_{0}=l \cos \phi+h \sin \phi \\
& h_{0}=-l \sin \phi+h \cos \phi
\end{aligned}
$$


The applied traction on the crack face, in order to eliminate the incident wave as indicated in (17), has the functional form $e^{p \lambda \bar{x}}$. Since the solution of applying traction $e^{p \eta \bar{x}}$ on crack faces has been solved in the previous section. The reflected and diffracted fields generated from the inclined crack can be constructed by superimposing the incident wave traction that is equal and opposite to (17). When we combine (6) and (17), the solution of reflected and diffracted waves for $\bar{\tau}_{\overline{y z}}$ and $\bar{\tau}_{\overline{x z}}$ in the Laplace transform domain can be expressed as follows:

$\tilde{\tau} \frac{d+r}{y z}(\bar{x}, \bar{y}, p)=\frac{1}{4 \pi^{2}} \int_{\Gamma_{\eta_{1}}} \int_{\Gamma_{\eta_{2}}} G\left(\eta_{1}, \eta_{2}\right) e^{-p\left(\alpha_{1} h_{0}+\eta_{1} l_{0}\right)} e^{-p\left(\alpha_{2} \bar{y}-\eta_{2} \bar{x}\right)} d \eta_{2} d \eta_{1}$

$\bar{\tau} \overline{x z}(\bar{x}, \bar{y}, p)=-\frac{1}{4 \pi^{2}} \int_{\Gamma_{\eta_{1}}} \int_{\Gamma_{\eta_{2}}} \frac{\eta_{2}}{\alpha_{2}} G\left(\eta_{1}, \eta_{2}\right) e^{-p\left(\alpha_{1} h_{0}+\eta_{1} l_{0}\right)} e^{-p\left(\alpha_{2} \bar{y}-\eta_{2} \bar{x}\right)} d \eta_{2} d \eta_{1}$

where

$G\left(\eta_{1}, \eta_{2}\right)=\frac{\left(b+\eta_{2}\right)^{1 / 2}}{\left(b+\eta_{1}\right)^{1 / 2}\left(\eta_{1}-\eta_{2}\right)}$

$\alpha_{1}=\left(b^{2}-\eta_{1}^{2}\right)^{1 / 2}$

$\alpha_{2}=\left(b^{2}-\eta_{2}^{2}\right)^{1 / 2}$

Since the dynamic stress intensity factor of applying traction $e^{p m \bar{x}}$ has been obtained in the previous section, the solution of the dynamic stress intensity factor due to the incident wave in the Laplace transform domain can be represented as follows

$\tilde{K}(p)=\frac{1}{2 \pi i} \int_{\Gamma_{\eta_{1}}} \frac{\left(\frac{2}{\pi}\right)^{1 / 2} \Gamma\left(\frac{1}{2}\right)}{\left(b+\eta_{1}\right)^{1 / 2} p^{1 / 2}} e^{-p\left(\alpha_{1} h_{0}+\eta_{1} t_{0}\right)} d \eta_{1}$

Eq. (22) constitutes an inversion integral where the path $\Gamma_{\eta_{1}}$ refers to Laplace inversion contour in the $\eta_{1}$ plane. The inverse transformation is carried out here through usage of the Cagniard-de Hoop technique. Cagniard contours are introduced here in $\eta_{1}$ plane by setting

$\alpha_{1} h_{0}+\eta_{1} l_{0}=t$

Eq. (23) can be solved for $\eta_{1}$ to yield

$\eta_{1}^{ \pm}=\frac{t \cos \Theta_{0}}{R_{0}} \pm i \frac{\sin \Theta_{0}}{R_{0}}\left(t^{2}-b^{2} R_{0}^{2}\right)^{1 / 2}$

where $\left(R_{0}, \Theta_{0}\right)=$ the polar coordinate of the source point and

$R_{0}=\left(l_{0}^{2}+h_{0}^{2}\right)^{1 / 2}$

$\Theta_{0}=\cos ^{-1}\left(\frac{l_{0}}{R_{0}}\right)$

In the $\eta_{1}$ plane, (24) describes a hyperbola that is denoted as the Cagniard contour. The $\Gamma_{\eta_{1}}$ integration is then shifted onto Cagniard contours along 
which $t$ is real and positive. By using the Cagniard-de Hoop method of the Laplace inversion, the dynamic stress intensity factor in time domain can be obtained as follows

$$
\begin{aligned}
& K(t)=\sqrt{\frac{2}{\pi^{3}}} \int_{b R_{0}}^{t} \operatorname{Im}\left[\frac{\frac{\partial \eta_{1}^{+}}{\partial \xi}}{\left(b+\eta_{1}^{+}\right)^{1 / 2}}\right] \frac{1}{(t-\xi)^{1 / 2}} d \xi \\
& =\sqrt{\frac{2}{\pi R_{0}}} \sin \left(\frac{\Theta_{0}}{2}\right) H\left(t-b R_{0}\right) \ldots \ldots \ldots \ldots \ldots
\end{aligned}
$$

The interesting result as shown in (26) is that the dynamic stress intensity factor jumps from zero to a static value after the incident shear wave generated from the loading point arrives at the crack tip. This static value is the correspondent solution of the stress intensity factor for applying static body force at $\bar{x}=l_{0}, \bar{y}=h_{0}$ and with twice the magnitude at an unbounded medium.

The incident shear wave ( $i$ wave) will generate a reflected wave ( $r$ wave) and diffracted wave ( $d$ wave) from the subsurface crack. After some later time, these two waves will reflect from the half-surface, these two reflected waves are indicated as the $r r$ wave and $d r$ wave. The solutions for $r r$ and $d r$ waves can be constructed by employing the method of images, which is easy to obtain from the solutions of $r$ and $d$ waves and the results are omitted here. The reflected $r r$ and $d r$ waves will arrive the crack tip at later time. The traction induced on the crack face by $r r$ and $d r$ waves in the Laplace transform domain can be expressed as follows

$\bar{\tau}_{\overline{y z}}(\bar{x}, 0, p)=\frac{1}{4 \pi^{2}} \int_{\Gamma_{\lambda_{1}}} \int_{\Gamma_{s_{22}}} G\left(\eta_{1}, \eta_{2}\right) e^{-p\left(\alpha_{1} h_{0}+\eta_{1} l_{0}\right)}$

$\cdot e^{-2 p h\left(\beta 22 \cos \phi+S_{22} \sin \phi\right)} e^{p S_{22} \bar{x}} d S_{22} d \eta_{1}$

where

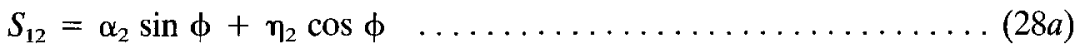

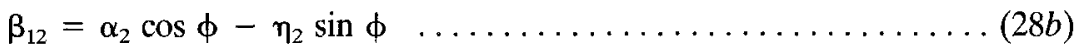

$S_{22}=\beta_{12} \sin \phi+S_{12} \cos \phi=\alpha_{2} \sin 2 \phi+\eta_{2} \cos 2 \phi \ldots \ldots \ldots \ldots(28 c)$

$\beta_{22}=\left(b^{2}-S_{22}^{2}\right)^{1 / 2}=\alpha_{2} \cos 2 \phi-\eta_{2} \sin 2 \phi \ldots \ldots \ldots \ldots \ldots \ldots(28 d)$

The applied traction on the crack face has the functional form of $e^{p S_{22 x}}$. Since the solutions of applying traction $e^{p \eta \bar{x}}$ on crack face have been previously solved. The dynamic stress intensity factor for the inclined crack due to the contribution of the $r r$ and $d r$ waves can be constructed by superimposing (10) and (27). The result for the dynamic stress intensity factor expressed in the Laplace transform domain is

$\bar{K}(p)=-\frac{\sqrt{\frac{2}{\pi}}}{4 \pi^{2}} \int_{\Gamma_{\eta_{1}}} \int_{\Gamma_{s_{22}}} \frac{\Gamma\left(\frac{1}{2}\right) G\left(\eta_{1}, \eta_{2}\right)}{p^{1 / 2}\left(b+S_{22}\right)^{1 / 2}} e^{-p\left(\alpha \mu_{1} h_{0}+\eta_{1} l_{0}\right)}$

$\cdot e^{-2 p h\left(\beta 22 \cos \phi+S_{22} \sin \phi\right)} d S_{22} d \eta_{1}$ 
The similar procedure is followed as previously indicated for constructing the contribution from the incident wave. Cagniard contours are introduced here by setting

$\alpha_{1} h_{0}+\eta_{1} l_{0}=t_{1}$

$2 h\left(\beta_{22} \cos \phi+S_{22} \sin \phi\right)=t_{2}$

which can be solved for $\eta_{1}$ and $S_{22}$ as follows

$\eta_{1}^{ \pm}=\frac{t_{1} \cos \Theta_{0}}{R_{0}} \pm i \frac{\sin \Theta_{0}}{R_{0}}\left(t_{1}^{2}-b^{2} R_{0}^{2}\right)^{1 / 2}$

$S_{22}^{ \pm}=\frac{t_{2} \sin \phi}{2 h} \pm i \frac{\cos \phi}{2 h}\left(t_{2}^{2}-4 b^{2} h^{2}\right)^{1 / 2}$

We shift the $\eta_{1}^{-}$and $S_{22}^{-}$integrations onto the associated Cagniard contours along which $t_{1}$ and $t_{2}$ are both real and positive. The two Cagniard contours must be superimposed in this technique for different locations of loading point and crack tip. Because $G\left(\eta_{1}, \eta_{2}\right)$ possesses a pole at $\eta_{1}=\eta_{2}$, the contribution of the pole has to be taken into account in the change of integral paths from $\eta_{1}$ to $t_{1}$ and $\eta_{2}$ to $t_{2}$. The contribution of this pole represents the fact that the reflected $r r$ wave would pass the crack tip and the condition for this to happen is $\Theta_{0}>\pi / 2+\phi$. Hence the contribution of the dynamic stress intensity factor due to $r r$ and $d r$ waves in time domain can be expressed as follows

$K(t)=\sqrt{\frac{2}{\pi R_{02}}} \sin \left(\frac{\Theta_{02}}{2}\right) H\left(t-b R_{02}\right)$

$-\frac{\sqrt{\frac{2}{\pi}}}{2 \pi^{2}} \int_{b R_{0}+2 b \hbar}^{t} \frac{1}{(t-u)^{1 / 2}} \int_{b R_{0}}^{u-2 b h} \operatorname{Re}\left[\frac{G\left(\eta_{1}^{+}, \eta_{2}^{+}\right)}{\left(b+S_{22}^{+}\right)^{1 / 2}} \frac{\partial \eta_{1}^{+}}{\partial t_{1}} \frac{\partial S_{22}^{+}}{\partial t_{2}}\right.$

$\left.-\frac{G\left(\eta_{1}^{-}, \eta_{2}^{+}\right)}{\left(b+S_{22}^{+}\right)^{1 / 2}} \frac{\partial \eta_{1}^{-}}{\partial t_{1}} \frac{\partial S_{22}^{+}}{\partial t_{2}} d t_{1}\right] d u \quad$ for $\Theta_{0}>\frac{\pi}{2}+\phi$

$K(t)=-\frac{\sqrt{\frac{2}{\pi}}}{2 \pi^{2}} \int_{b R_{0}+2 b h}^{t} \frac{1}{(t-u)^{1 / 2}} \int_{b R_{0}}^{u-2 b h} \operatorname{Re}\left[\frac{G\left(\eta_{1}^{+}, \eta_{2}^{+}\right)}{\left(b+S_{22}^{+}\right)^{1 / 2}} \frac{\partial \eta_{1}^{+}}{\partial t_{1}} \frac{\partial S_{22}^{+}}{\partial t_{2}}\right.$

$\left.-\frac{G\left(\eta_{1}^{-}, \eta_{2}^{+}\right)}{\left(b+S_{22}^{+}\right)^{1 / 2}} \frac{\partial \eta_{1}^{-}}{\partial t_{1}} \frac{\partial S_{22}^{+}}{\partial t_{2}} d t_{1}\right] d u \quad$ for $\Theta_{0}<\frac{\pi}{2}+\phi$

where

$R_{02}=\left(l_{02}^{2}+h_{02}^{2}\right)^{1 / 2}$

$\Theta_{02}=\cos ^{-1}\left(\frac{l_{02}}{R_{02}}\right)$

$l_{02}=l \cos 3 \phi+h \sin 3 \phi+2 h \sin \phi$ 
$h_{02}=-l \sin 3 \phi+h \cos 3 \phi+2 h \cos \phi$

$t_{2}=t-t_{1}$

The arrival times of the wave fronts for $r r$ and $d r$ waves are found to be $b R_{02}$ and $b\left(2 h+R_{0}\right)$, respectively. The contribution of the $r r$ wave for the dynamic stress intensity factor is expressed in the first term of (32). It is also very interesting to find that this value is time-independent and has the same feature as the contribution from the incident wave.

Follow the similar procedure that used for constructing the dynamic stress intensity factor for the first few waves. The complete transient solution of the dynamic stress intensity factor is finally obtained explicitly. The image method shown in Fig. 2 is used for constructing the solution. The complete solution for dynamic stress intensity factor can be simplified into a very compact form as follows

$$
K(t)=\sum_{k=0}^{n} K^{k}(t)+\sum_{k=0}^{n} \sum_{l=1}^{\infty} K^{k, l}(t)
$$

The complete solutions consist of two terms as shown in (35). The first term with one summation is the contributions for incident wave and reflected waves, which are only generated by purely reflected waves, i.e., $k=0$ for incident wave, $k=1$ for $r r$ wave, $k=2$ for $r r r$ wave. The explicit forms are expressed as follows

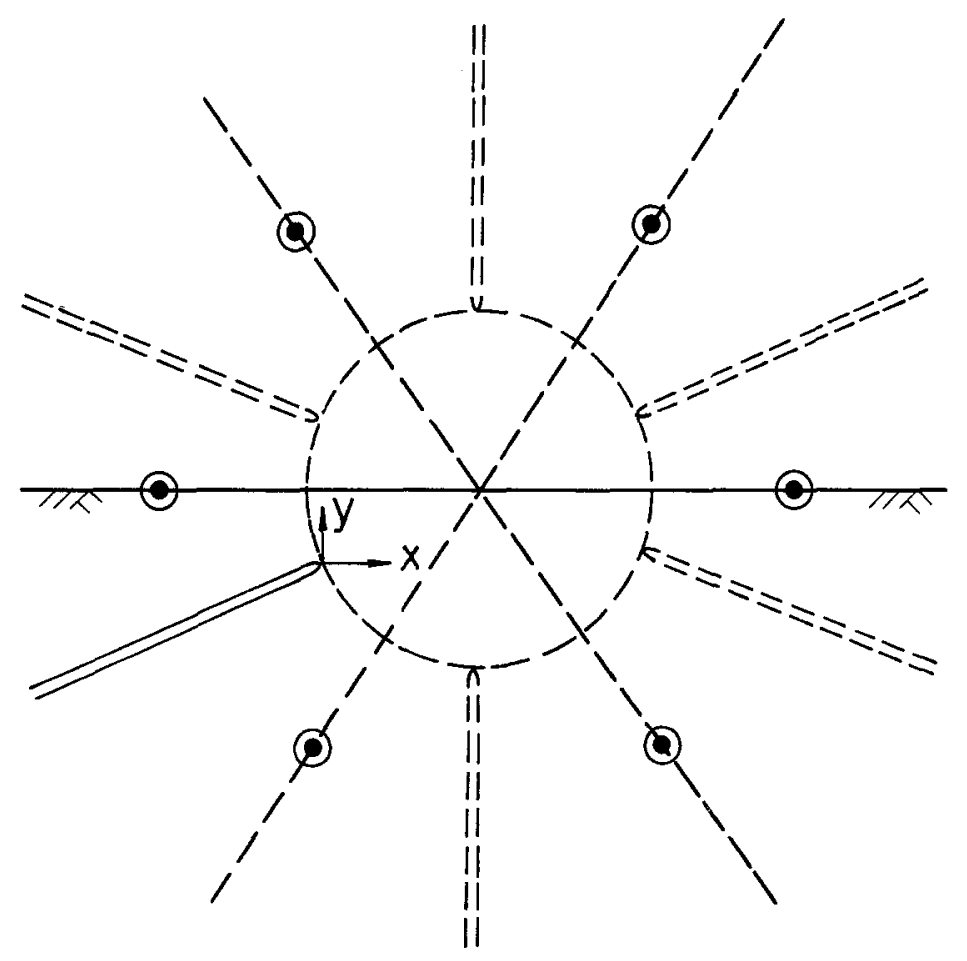

FIG. 2. Image Method Used to Construct Transient Solutions 
$K^{k}(t)=\sqrt{\frac{2}{\pi R_{0,2 k}}} \sin \left(\frac{\Theta_{0,2 k}}{2}\right) H\left(t-b R_{0,2 k}\right)$

where

$$
\begin{aligned}
& R_{0,2 k}=\left(l_{0,2 k}^{2}+h_{0,2 k}^{2}\right)^{1 / 2} \ldots \ldots \ldots \ldots \ldots \ldots \ldots \ldots \ldots \ldots \ldots \ldots \ldots \ldots \ldots \ldots \ldots \ldots \ldots \ldots \ldots \ldots \ldots \ldots \ldots \ldots \ldots \ldots \ldots \ldots \\
& \cos \Theta_{0,2 k}=\frac{l_{0,2 k}}{R_{0,2 k}} \ldots \ldots \ldots \ldots \ldots \ldots \ldots \\
& l_{0,2 k}=l \cos (2 k+1) \phi+h \sin (2 k+1) \phi+\sum_{m=0}^{k-1} 2 h \sin (2 m+1) \phi .(37 \\
& h_{0,2 k}=-l \sin (2 k+1) \phi+h \cos (2 k+1) \phi+\sum_{m=0}^{k-1} 2 h \cos (2 m+1) \phi
\end{aligned}
$$

It is shown from the above expression that the contribution of each pure reflected wave on the stress intensity factor is a finite jump and is timeindependent.

The second term in (35) with two summations comes from the contributions for reflected waves from half-plane surface, which are generated by diffracted waves from the crack tip, i.e., $k=0, l=1$ for $d r$ wave, $l=2$ for $d r d r$ wave, $l=3$ for $d r d r d r$ wave; $k=1, l=1$ for $r r d r$ wave, $l=2$ for $r r d r d r$ wave; $l=3$ for $r r d r d r d r$ wave. $k=0$ can be seen here to consist of diffracted $d$ wave, which is diffracted by the incident wave and infinite sequence of reflected waves (i.e., $d r$ wave, $d r d r$ wave, and so forth), which is generated by the $d$ wave. $k=1$ consists of diffracted $r r d$ wave, which is diffracted by the crack tip for $r r$ wave and infinite sequence of reflected waves (i.e., $r r d r$ wave, $r r d r d r$ wave, and so forth), which is generated by the $r r d$ wave. The results of the dynamic stress intensity factor due to the contribution of each wave indicated above can be expressed as follows

$$
\begin{gathered}
K^{k, l}(t)=\frac{-\sqrt{\frac{2}{\pi}}(i)^{q}}{2 \pi^{2}(2 \pi i)^{l-1}} \int_{b R_{0,2 k+2 h b b}}^{t} \frac{1}{(t-u)^{1 / 2}} \\
\cdot \int_{b R_{0,2 k}}^{b_{1}} \int_{2 h b}^{b_{2}} \cdots \int_{2 h b}^{b_{l}} F U N K_{k_{l} l} d t_{l} d t_{l-1} \cdots d t_{1} d u
\end{gathered}
$$

where

$$
\begin{aligned}
& F U N K_{k, l}=\operatorname{Re}\left\{\left[\frac{1}{\left(b^{2}+S_{2, k+l+1}^{+1 / 2}\right)^{1 / 2}}\right] G\left(S_{\frac{ \pm}{2, k+1}}^{ \pm}, \eta_{\bar{k}+2}^{ \pm}\right)\right. \\
& G\left(S_{\overline{2}, k+2}^{ \pm}, \eta_{\bar{k}+3}^{ \pm}\right) \cdots G\left(S_{2, k+l}^{ \pm}, \eta_{k+l+1}^{+}\right)\left( \pm \frac{\partial S_{2, k+1}^{ \pm}}{\partial t_{k+1}}\right) \\
& \left.\cdot\left( \pm \frac{\partial S_{2, k+2}^{ \pm}}{\partial t_{k+2}}\right) \cdots\left( \pm \frac{\partial S_{2, k+1}^{ \pm}}{\partial t_{k+1}}\right)\left(\frac{\partial S_{2, k+l+1}^{+}}{\partial t_{k+l+1}}\right)\right\} \quad \text { for } l=1,3,5, \ldots
\end{aligned}
$$




$$
\begin{aligned}
& F U N K_{k, l}=\operatorname{Im}\left\{\left[\frac{1}{\left(b^{2}+S_{2, k+l+1}^{+}\right)^{1 / 2}}\right] G\left(S_{2, k+1}^{ \pm}, \eta_{\frac{ \pm}{k+2}}^{+}\right)\right. \\
& \cdot G\left(S_{2, k+2}^{ \pm}, \eta_{\bar{k}+3}^{ \pm}\right) \cdots G\left(S_{2, k+l}^{ \pm}, \eta_{k+I+1}^{+}\right)\left( \pm \frac{\partial S_{2, k+1}^{ \pm}}{\partial t_{k+1}}\right) \\
& \left.\cdot\left( \pm \frac{\partial S_{2, k+2}^{ \pm}}{\partial t_{k+2}}\right) \ldots\left( \pm \frac{\partial S_{2, k+l}^{ \pm}}{\partial t_{k+1}}\right)\left(\frac{\partial S_{2, k+l+1}^{+}}{\partial t_{k+l+1}}\right)\right\} \quad \text { for } l=2,4,6, \ldots
\end{aligned}
$$

in which

$$
\begin{aligned}
& S_{2, k+1}^{ \pm}=\frac{t_{k+1} \cos \Theta_{0,2 k}}{R_{0,2 k}} \pm i \frac{\sin \Theta_{0,2 k}}{R_{0,2 k}}\left(t_{k+1}^{2}-b^{2} R_{0,2 k}^{2}\right)^{1 / 2} \\
& S_{2, k+z^{\prime}+1}^{ \pm}=\frac{t_{k+z^{\prime}+1} \sin \phi}{2 h} \pm i \frac{\cos \phi}{2 h}\left(t_{k+v^{\prime}+1}^{2}-4 b^{2} h^{2}\right)^{1 / 2} \\
& (v=1,2,3, \ldots, l) \\
& \eta_{k+i+1}^{ \pm}=-\frac{t_{k+v+1} \sin \phi}{2 h} \pm i \frac{\cos \phi}{2 h}\left(t_{k+v+1}^{2}-4 b^{2} h^{2}\right)^{1 / 2} \\
& (v=1,2,3, \ldots, l) \\
& q=1 \quad \text { when } l=0,2,4,6, \ldots ; \quad q=0 \\
& \text { when } l=1,3,5,7, \ldots \\
& b_{1}=u-2 l h b, b_{m+1}=u-t_{1}-t_{2} \cdots-t_{m}-2(l-m) h b \\
& (m=1,2, \ldots, l-1)
\end{aligned}
$$

The number of purely reflected waves (i.e., $n$ ) that will pass through the crack tip and generate infinite series of diffracted wave is dependent on the location and the inclined angle $\phi$ of the crack, and the position where the dynamic point loading is applied. The result is expressed as follows

$$
\text { If } \phi_{2 j+1}<\frac{\pi}{2}<\phi_{2 j-1} \text {, then } n=j
$$

where

$$
\begin{aligned}
& \phi_{2 j+1}=\cos ^{-1}\left(\frac{l_{2 j+1}}{R_{2 j+1}}\right) \\
& R_{2 j+1}=\left(l_{2 j+1}^{2}+h_{2 j+1}^{2}\right)^{1 / 2} \\
& l_{2 j+1}=l \cos 2(j+1) \phi+h \sin 2(j+1) \phi+\sum_{p=1}^{j} 2 h \sin (2 p \phi) \\
& h_{2 j+1}=l \sin 2(j+1) \phi-h \cos 2(j+1) \phi-\sum_{p=1}^{j} 2 h \cos (2 p \phi)
\end{aligned}
$$




\section{NUMERICAL RESULTS OF TRANSIENT ANALYSIS}

The geometric configuration considered in this study is an inclined semiinfinite crack located under the surface of a half-plane. The incident wave generated by the dynamic antiplane loading will be diffracted from the crack tip and reflected from the crack surface as $d$ and $r$ waves, which will be reflected from the half-plane as $d r$ and $r r$ wave and interact with the inclined crack again at later time. The structure of the wave fronts for incident, reflected, and diffracted waves for a short time period is expressed in Fig. 3.

\section{O}

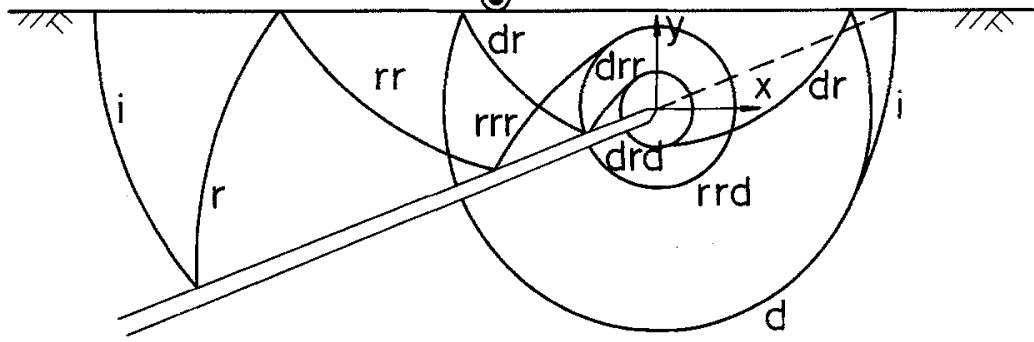

FIG. 3. Wave Fronts of Incident, Reflected, and Diffracted Waves for Short Time Period after Impact Loading Is Applied on Half-Space

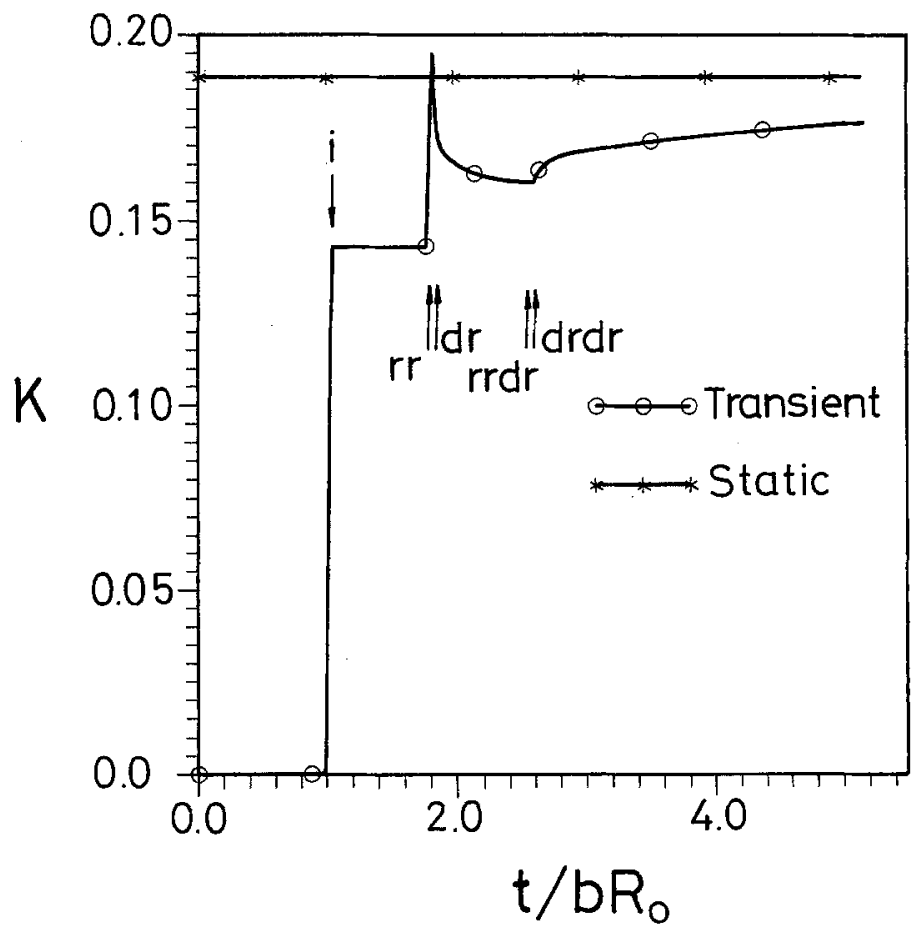

FIG. 4. Transient Response of Dynamic Stress Intensity Factor Due to Impact Loading Applied at $(-25,10)$ for Inclined Angle $\phi=30^{\circ}$ 


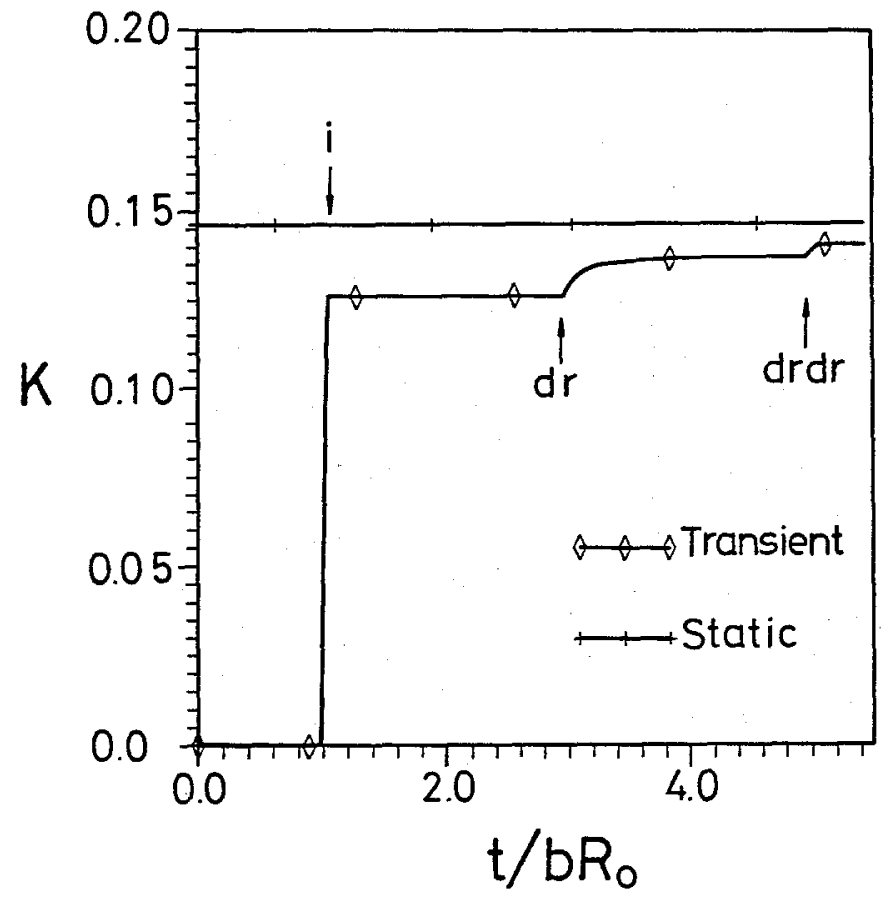

FIG. 5. Transient Response of Dynamic Stress Intensity Factor Due to Impact Loading Applied at $(0,10)$ for Inclined Angle $\phi=30^{\circ}$

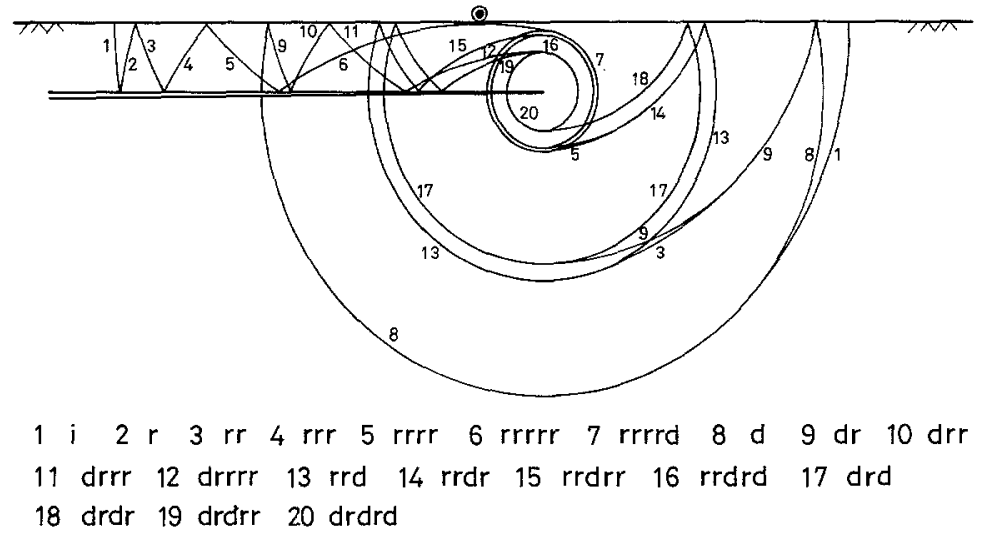

FIG. 6. Wave Fronts of Incident, Reflected, and Diffracted Waves for Long Time Period after Dynamic Loading Is Applied on Half-Space

In the previous section, the exact transient elastodynamic stress intensity factor has been determined. The transient response of stress intensity factor is investigated here for a point dynamic loading with a Heaviside function $H(t)$ time dependence applied at $(-25,10)$. The applied loading is located at the left-hand side of the crack tip and the inclined angle $\phi$ of crack is chosen to be $30^{\circ}$. It is worth noting that only the incident wave and the 


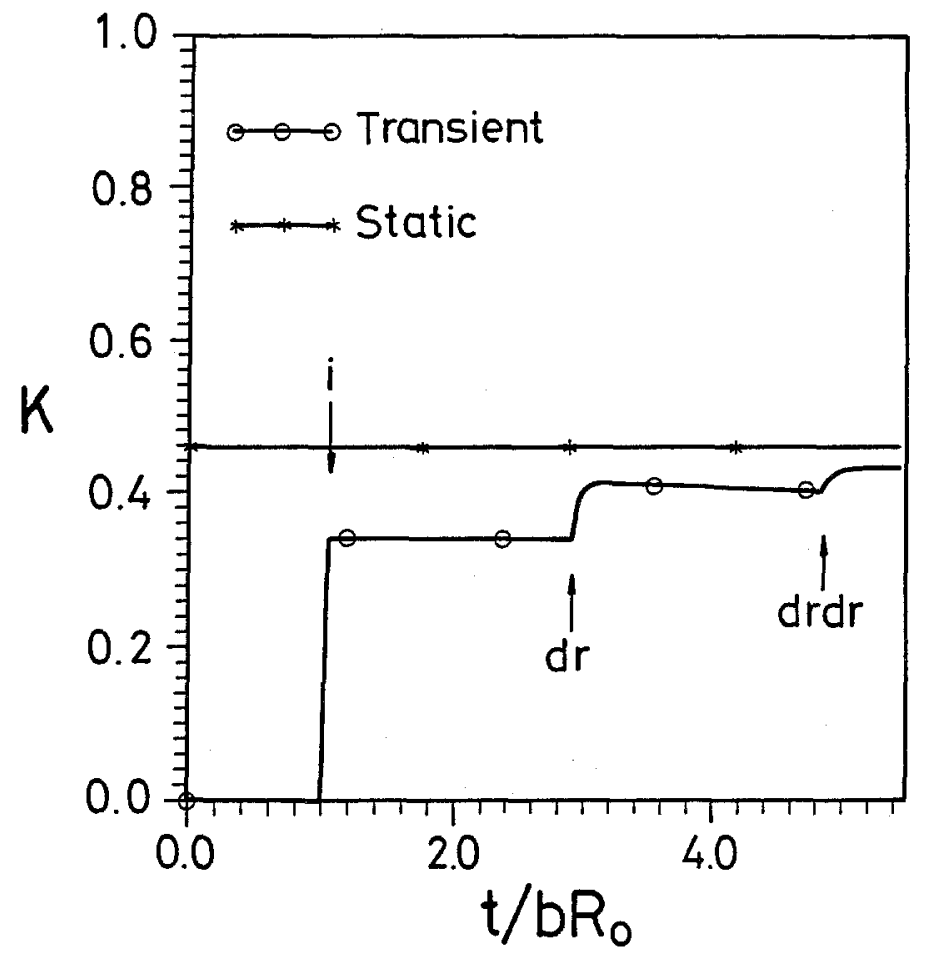

FIG. 7. Transient Response of Dynamic Stress Intensity Factor Due to Impact Loading Applied at $(0.5,2)$ for Crack Parallel to Half-Space

reflected $r r$ wave will pass through the crack tip and generate a sequence of diffracted waves; the other pure reflected waves (i.e., $r r r$ wave and rrrrr wave, and so forth) will only reflect back and forth between the crack face and the half-plane surface. Hence obtained here is $k$ equal to 0 and 1 (or $n=1$ ) in this particular case, and the associated wave fronts for incident, reflected, and diffracted waves for a short time period are shown in Fig. 3. The transient response of the dynamic stress intensity factor is shown in Fig. 4 . The time has been normalized by dividing $b R_{0}$, where $R_{0}=$ the distance from the point loading to the crack tip. The corresponding static value is also indicated in the figures. The normalized arrival times for the first few wave fronts at the crack tip are 1 for the incident $i$ wave, 1.738 for the $r r$ wave, 1.742 for the $d r$ wave, and 2.48 for the $r r d r$ wave. The history of stress intensity factor shows a finite jump as the $i$ wave and $r r$ wave have arrived the crack tip. The transient solution of the stress intensity factor will tend toward the corresponding static value after the second diffracted wave has passed the crack tip. Next, we consider the point loading being applied directly above the crack tip, so that $l=0$ and $h=10$. In this case, only incident wave will generate a sequence of diffracted waves, hence $n$ $=0$. The transient result of the stress intensity factor is plotted in Fig. 5 . The normalized arrival times are 1 for the $i$ wave, 3 for the $d r$ wave, and 5 for the $d r d r$ wave. It is also indicated in this figure that after the second diffracted wave has passed, the transient solution of the dynamic stress intensity factor will approach toward the static value. 
Finally, we consider the case that the crack is parallel to the half plane surface, i.e., $\phi=0$. For applying point loading at the left hand side of the crack tip, the complicated wave fronts generated from the crack and the half plane surface for quite a long time are shown in Fig. 6. For the numerical investigation, we consider a dynamic point loading with a Heaviside function time dependence is applied at $(0.5,2)$, which is at the right-hand side of the crack tip. In this case, only incident wave will generate a sequence of diffracted waves, hence $n=0$. The transient history of the dynamic stress intensity factor is shown in Fig. 7. The normalized arrival times for the wave fronts are 1 for the $i$ wave, 2.94 for the $d r$ wave, and 4.88 for the $d r d r$ wave. It is also shown in this figure that after the $d r d r$ wave has passed the crack tip, the transient solution will approach toward the static value.

\section{CONCLUSIONS}

Most of the problems that have been studied in the development of fracture mechanics are quasi-static. Because of loading conditions and material properties, numerous problems have existed for which the assumption that the deformation is quasi-static is invalid and the inertia of the material must be taken into account. The propagation of stress waves through an unbounded medium is not a difficult subject. However, if boundaries are introduced, reflected and diffracted waves will be generated from boundaries, making the problem much more complicated.

The transient response of a half-space containing a subsurface inclined crack has been considered here to gain insight into understanding the interaction of stress waves with material defects. This problem contains a characteristic length and is solved by superposition of the fundamental solutions in the Laplace transform domain. This new methodology is shown to be both powerful and efficient in solving more difficult problems. The exact transient solutions of dynamic stress intensity factor for a long period of time are obtained in this study. The closed-form transient solution is expressed in a very simple formulation that accounts for all the contributions coming from incident, reflected, and diffracted waves. The solution of dynamic stress intensity factor obtained in this study can also be used for analyzing the condition for the unstable crack propagation under dynamic loading.

One of the main objectives in this study is to investigate the characteristic time that the transient solution would approach toward the correspondent static value in dynamic fracture problems. With the exact analytic solutions at hand, numerical calculations for the dynamic stress intensity factor have been presented and compared to corresponding static values. It is found in this study that the transient effect in this problem can be neglected after the first few waves have passed the crack tip.

\section{ACKNOWLEDGMENTS}

The research support of the National Science Council, Republic of China, through Grant NSC 81-0401-E002-18 at National Taiwan University is gratefully acknowledged.

\section{APPENDIX I. REFERENCES}

Boström, A. (1987). "Elastic wave scattering from an interface crack: antiplane strain." J. Appl. Mech. Trans. ASME, 54(3), 503-508. 
Brock, L. M. (1982). "Shear and normal impact loadings on one face of a narrow slit." Int. J. Solids Struct., 18(6), 467-477.

Brock, L. M. (1983). "The dynamic stress intensity factor due to arbitrary screw dislocation motion." J. Appl. Mech. Trans. ASME, 50(2), 383-389.

Brock, L. M. (1984). "Stresses in a surface obstacle undercut due to rapid indentation." J. Elast., 14, 415-424.

Brock, L. M. (1986). "Transient dynamic green's functions for a cracked plane." $Q$. Appl. Math., 44(2), 265-275.

Brock, L. M., Jolles, M, and Schroedl, M. (1985). "Dynamic impact over a subsurface crack: applications to the dynamic tear test." J. Appl. Mech. Trans. ASME, 52(2), 287-290.

de Hoop, A. T. (1958). "Representation theorems for the displacement in an elastic solid and their application to elastodynamic diffraction theory," $\mathrm{PhD}$ thesis, Technische Hoegschool, Delft, Netherlands.

Freund, L. B. (1974). "The stress intensity factor due to normal impact loading of the faces of a crack." Int. J. Eng. Sci., 12, 179-189.

Kundu, T. (1986). "Transient response of an interface-crack in a layered plate." $J$. Appl. Mech. Trans. ASME, 53(3), 579-586.

Lee, Y. J., and Freund, L. B. (1990). "Fracture initiation due to asymmetric impact loading of an edge cracked plate." J. Appl. Mech. Trans. ASME, 57(1), 104-111.

Ma, C. C., and Chen, S. K. (1993). "Exact transient analysis of an anti-plane semiinfinite crack subjected to dynamic body forces." Wave Motion, 17(2), 161-171.

Ma, C. C., and Hou, Y. C. (1990). "Theoretical analysis of the transient response for a stationary inplane crack subjected to dynamic impact loading." Int. J. Eng. Sci., 28(12), 1321-1329.

Ma, C. C., and Hou, Y. C. (1991). "Transient analysis for antiplane crack subjected to dynamic loadings." J. Appl. Mech. Trans. ASME, 58(3), 703-709.

Noble, B. (1958). The wiener-hopf technique, Pergamon Press, Elmsford, N.Y.

Srivastava, K. N., Gupta, O. P., and Palaiya, R. M. (1978). "Interaction of elastic waves in two bonded dissimilar elastic half-planes having Griffith crack at interfaceI." Int. J. Fracture, 14(4), 145-154.

Tsai, C. H., and Ma, C. C. (1992). "Transient analysis of a semi-infinite crack subjected to dynamic concentrated forces." J. Appl. Mech. Trans. ASME, 59(4), 804-811.

Tsai, C. H., and Ma, C. C. (1993). "The stress intensity factor of a subsurface inclined crack subjected to dynamic impact loading." Int. J. Solids Struct., 30(16), 2163-2175.

Yang, H. J., and Bogy, D. B. (1985). "Elastic wave scattering from an interface crack in a layered half space." J. Appl. Mech. Trans. ASME, 52(1), 42-50.

\section{APPENDIX II. NOTATION}

The following symbols are used in this paper:

$b=$ slowness of shear wave;

$h=$ vertical distance from point loading to crack tip;

$K=$ dynamic stress intensity factor;

$l=$ horizontal distance from point loading to crack tip;

$t=$ time;

$w=$ displacement;

$x, y=$ Cartesian coordinate;

$\mu=$ shear modulus;

$\rho=$ mass density; and

$\phi=$ inclined angle of crack. 